PROCEEDINGS OF THE

AMERICAN MATHEMATICAL SOCIETY

Volume 136, Number 7, July 2008, Pages 2359-2363

S 0002-9939(08)09260-5

Article electronically published on March 4, 2008

\title{
ON THE COFINITENESS OF LOCAL COHOMOLOGY MODULES
}

\author{
KAMAL BAHMANPOUR AND REZA NAGHIPOUR
}

(Communicated by Bernd Ulrich)

\begin{abstract}
In this note we show that if $I$ is an ideal of a Noetherian ring $R$ and $M$ is a finitely generated $R$-module, then for any minimax submodule $N$ of $H_{I}^{t}(M)$ the $R$-module $\operatorname{Hom}_{R}\left(R / I, H_{I}^{t}(M) / N\right)$ is finitely generated, whenever the modules $H_{I}^{0}(M), H_{I}^{1}(M), \ldots, H_{I}^{t-1}(M)$ are minimax. As a consequence, it follows that the associated primes of $H_{I}^{t}(M) / N$ are finite. This generalizes the main result of Brodmann and Lashgari (2000).
\end{abstract}

\section{INTRODUCTION}

It is well known that for a Noetherian ring $R$, an ideal $I$ of $R$, and a finitely generated $R$-module $M$, the local cohomology modules $H_{I}^{i}(M)$ are not always finitely generated. On the other hand if $(R, \mathfrak{m})$ is a local ring with residue field $k$, then the local cohomology modules $H_{\mathfrak{m}}^{i}(M)$ are Artinian, so $\operatorname{Hom}_{R}\left(k, H_{\mathfrak{m}}^{i}(M)\right)$ is finitely generated. Taking this fact, Grothendieck [6] conjectured the following:

If $R$ is a Noetherian ring, then for any ideal $I$ of $R$ and any finitely generated $R$-module $M$, the module $\operatorname{Hom}_{R}\left(R / I, H_{I}^{i}(M)\right)$ is finitely generated.

Here, $H_{I}^{j}(M)$ denotes the $j^{\text {th }}$ local cohomology module of $M$ with support in I. This conjecture is false in general. In fact, Hartshorne [7 gave the following counterexample:

Let $k$ be a field and let $R=k[x, y, z, u] /(x y-z u)$. Set $I=(x, u)$. Then

$$
\operatorname{Hom}_{R}\left(R / I, H_{I}^{2}(R)\right)
$$

is not finitely generated.

On the other hand, an important problem in commutative algebra is determining when the set of associated primes of the $i^{\text {th }}$ local cohomology modules $H_{I}^{i}(M)$ of $M$ with support in $I$ is finite (see [9, Problem 4]). A. Singh [18] and M. Katzman [11 have given counterexamples to this conjecture. However, it is known that this conjecture is true in many situations; see, [1, 2], 8], 10, 12, 14. In particular, Brodmann and Lashgari [1, Theorem 2.2] showed that, if for a finitely generated $R$-module $M$ and an integer $t$, the local cohomology modules $H_{I}^{0}(M), H_{I}^{1}(M), \ldots, H_{I}^{t-1}(M)$ are finitely generated, then $\operatorname{Ass}_{R}\left(H_{I}^{t}(M) / N\right)$ is finite

Received by the editors February 27, 2007, and, in revised form, May 17, 2007.

2000 Mathematics Subject Classification. Primary 13D45, 14B15, 13 E05.

Key words and phrases. Local cohomology, cofinite module, minimax module, associated primes.

The research of the second author has been in part supported by a grant from IPM (No. 85130042).

(C)2008 American Mathematical Society Reverts to public domain 28 years from publication 
for every finitely generated submodule $N$ of $H_{I}^{t}(M)$. For a survey of recent developments on finiteness properties of local cohomology modules, see Lyubeznik's interesting paper [13]. This paper is concerned with what might be considered a generalization of the above mentioned result of Brodmann and Lashgari to the class of minimax modules. More precisely, we shall show that:

Theorem 1.1. Let $R$ be a Noetherian ring and $I$ an ideal of $R$. Let $M$ be a nonzero finitely generated $R$-module and let $t \geq 0$ be an integer such that $H_{I}^{i}(M)$ is a minimax $R$-module for all $i<t$. Then, for every minimax submodule $N$ of $H_{I}^{t}(M)$, the $R$-module $\operatorname{Hom}_{R}\left(R / I, H_{I}^{t}(M) / N\right)$ is finitely generated.

Recall that a module is called minimax module when it has a finitely generated submodule, such that the quotient by it is an Artinian module [20.

One of our tools for proving Theorem 1.1 is the following:

Lemma 1.2. Let $I$ denote an ideal of a Noetherian ring $R$ and let $M$ be a nonzero finitely generated $R$-module. Let $t \geq 0$ be an integer such that $H_{I}^{i}(M)$ is $I$-cofinite and minimax $R$-module for all $i<t$. Then $\operatorname{Hom}_{R}\left(R / I, H_{I}^{t}(M)\right)$ is a finitely generated $R$-module.

Throughout this paper, $R$ will always be a commutative Noetherian ring with non-zero identity, $M$ will be a non-zero finitely generated $R$-module, and $I$ will be an ideal of $R$. The $i^{\text {th }}$ local cohomology module of $M$ with support in $I$ is defined by

$$
H_{I}^{i}(M)=\underset{n \geq 1}{\lim _{n}} \operatorname{Ext}_{R}^{i}\left(R / I^{n}, M\right) .
$$

We refer the reader to [5] or [3] for the basic properties of local cohomology.

\section{The RESUlts}

Let us, first, recall the important concept of an $I$-cofinite of an $R$-module $N$ with respect to an ideal $I$ of $R$, introduced by Hartshorne in [7. For any ideal $I$ of $R$, Hartshorne defined a module $N$ to be $I$-cofinite, if $\operatorname{Supp}(N) \subseteq V(I)$ and $\operatorname{Ext}_{R}^{i}(R / I, N)$ is finitely generated module for all $i$.

In [20] $\mathrm{H}$. Zöschinger introduced the interesting class of minimax modules, and he has in 20, 21, given many equivalent conditions for a module to be minimax. The $R$-module $N$ is said to be a minimax module, if there is a finitely generated submodule $L$ of $N$, such that $N / L$ is Artinian. The class of minimax modules thus includes all finitely generated and all Artinian modules. It was shown by T. Zink 19 and by E. Enochs [4 that a module over a complete local ring is minimax if and only if it is Matlis reflexive.

The following lemma is needed in the proof of main theorem.

Lemma 2.1. Let $R$ be a Noetherian ring and let $0 \longrightarrow N^{\prime} \longrightarrow N \longrightarrow N^{\prime \prime} \longrightarrow 0$ be an exact sequence of $R$-modules. Then $N$ is minimax if and only if $N^{\prime}$ and $N^{\prime \prime}$ are both minimax.

Proof. We may suppose for the proof that $N^{\prime}$ is a submodule of $N$ and that $N^{\prime \prime}=$ $N / N^{\prime}$. If $N$ is minimax, then it easily follows from the definition that $N^{\prime}$ and $N / N^{\prime}$ are minimax. Now, suppose that $N^{\prime}$ and $N / N^{\prime}$ are minimax. Then there exists a finitely generated submodule $T$ of $N^{\prime}$, such that $N^{\prime} / T$ is Artinian. Let $M^{\prime}=N^{\prime} / T$ and $M=N / T$. Then we obtain the exact sequence

$$
0 \longrightarrow M^{\prime} \longrightarrow M \longrightarrow M / M^{\prime} \longrightarrow 0
$$


where $M^{\prime}$ is Artinian and $M / M^{\prime}$ is minimax (note that $M / M^{\prime} \cong N / N^{\prime}$ ). Now, since $M / M^{\prime}$ is minimax it follows from the definition that there is a finitely generated submodule $L / M^{\prime}$ of $M / M^{\prime}$ such that $M / L$ is Artinian. As $L / M^{\prime}$ is finitely generated, it follows that $L=M^{\prime}+K$ for some finitely generated submodule $K$ of $L$. Then it follows from $L / K \cong M^{\prime} / K \cap M^{\prime}$ that $L / K$ is an Artinian $R$-module. Therefore the exact sequence

$$
0 \longrightarrow L / K \longrightarrow M / K \longrightarrow M / L \longrightarrow 0
$$

implies that $M / K$ is Artinian. Consequently $M$ is a minimax module. Since $M=N / T$, it follows that $K=S / T$ for some submodule $S$ of $N$. As $T$ and $K$ are finitely generated, we deduce that $S$ is also finitely generated. Now because

$$
N / S \cong(N / T) /(S / T)=M / K
$$

is Artinian, we get from definition that $N$ is minimax and the claim is true.

The following lemma plays a key role in the proof of the main theorem.

Lemma 2.2. Let $R$ be a Noetherian ring, $M$ a non-zero finitely generated $R$ module and $I$ an ideal of $R$. Let $t$ be a non-negative integer, such that $H_{I}^{i}(M)$ is $I$-cofinite minimax for all $i<t$. Then, the $R$-module $\operatorname{Hom}_{R}\left(R / I, H_{I}^{t}(M)\right)$ is finitely generated. In particular, the set $\operatorname{Ass}_{R}\left(H_{I}^{t}(M)\right)$ is finite.

Proof. We use induction on $t$. When $t=0$ there is nothing to prove. So suppose that $t>0$ and that the result has been proved for smaller values of $t$. It follows from [3, Corollary 2.1.7] that $H_{I}^{i}(M) \cong H_{I}^{i}\left(M / \Gamma_{I}(M)\right)$ for all $i>0$. Also, by [3, Lemma 2.1.2], $M / \Gamma_{I}(M)$ is an $I$-torsion-free $R$-module. Hence we can (and do) assume that $M$ is an $I$-torsion-free $R$-module. Then, in view of [3, Lemma 2.1.1], the ideal $I$ contains an element $x$ which is $M$-regular. The exact sequence

$$
0 \longrightarrow M \stackrel{x}{\longrightarrow} M \longrightarrow M / x M \longrightarrow 0
$$

induces a long exact sequence

$$
\cdots \longrightarrow H_{I}^{i}(M) \stackrel{x}{\longrightarrow} H_{I}^{i}(M) \longrightarrow H_{I}^{i}(M / x M) \longrightarrow H_{I}^{i+1}(M) \stackrel{x}{\longrightarrow} H_{I}^{i+1}(M) \longrightarrow \cdots
$$

Therefore we deduce that the sequence

$$
0 \longrightarrow H_{I}^{i-1}(M) / x H_{I}^{i-1}(M) \longrightarrow H_{I}^{i-1}(M / x M) \longrightarrow\left(0:_{H_{I}^{i}(M)} x\right) \longrightarrow 0
$$

is exact. Also, by [16. Corollary 4.4], Lemma 2.1 and the hypothesis, $H_{I}^{i-1}(M / x M)$ is $I$-cofinite minimax for all $i<t$, so that, by the inductive hypothesis,

$$
\operatorname{Hom}_{R}\left(R / I, H_{I}^{t-1}(M / x M)\right)
$$

is finitely generated. On the other hand, the exact sequence

$$
0 \longrightarrow H_{I}^{t-1}(M) / x H_{I}^{t-1}(M) \longrightarrow H_{I}^{t-1}(M / x M) \longrightarrow\left(0:_{H_{I}^{t}(M)} x\right) \longrightarrow 0
$$

induces the exact sequence

$$
\begin{aligned}
\operatorname{Hom}_{R}\left(R / I, H_{I}^{t-1}(M / x M)\right) & \longrightarrow \operatorname{Hom}_{R}\left(R / I, 0:_{H_{I}^{t}(M)} x\right) \\
& \longrightarrow \operatorname{Ext}_{R}^{1}\left(R / I, H_{I}^{t-1}(M) / x H_{I}^{t-1}(M)\right) .
\end{aligned}
$$

Since by [16, Corollary 4.4] $H_{I}^{t-1}(M) / x H_{I}^{t-1}(M)$ is $I$-cofinite minimax, it follows from the above exact sequence that $\operatorname{Hom}_{R}\left(R / I, 0:_{H_{I}^{t}(M)} x\right)$ is finitely generated. Now, as $x \in I$ we have

$\operatorname{Hom}_{R}\left(R / I, 0:_{H_{I}^{t}(M)} x\right) \cong \operatorname{Hom}_{R}\left(R / I \otimes_{R} R /(x), H_{I}^{t}(M)\right) \cong \operatorname{Hom}_{R}\left(R / I, H_{I}^{t}(M)\right)$, 
and so $\operatorname{Hom}_{R}\left(R / I, H_{I}^{t}(M)\right)$ is finitely generated. This completes the inductive step.

The following result will be used for the generalization of the main result of Nhan and Brodmann-Lashgari.

Theorem 2.3. Let $R$ be a Noetherian ring, $M$ a non-zero finitely generated $R$ module and $I$ an ideal of $R$. Let $t$ be a non-negative integer such that $H_{I}^{i}(M)$ is minimax for all $i<t$. Then the $R$-module $\operatorname{Hom}_{R}\left(R / I, H_{I}^{t}(M)\right)$ is finitely generated. In particular the set $\operatorname{Ass}_{R}\left(H_{I}^{t}(M)\right)$ is finite.

Proof. In view of Lemma 2.2 it is enough for us to show that $H_{I}^{i}(M)$ is $I$-cofinite for all $i<t$. We proceed by induction on $i$. The case $i=0$ is obvious as $H_{I}^{0}(M)$ is finitely generated. So, let $i>0$, and the result has been proved for smaller values of $i$. By the inductive assumption, $H_{I}^{j}(M)$ is $I$-cofinite for $j=0,1, \ldots, i-1$. Hence by Lemma 2.2 and assumption, $\operatorname{Hom}_{R}\left(R / I, H_{I}^{i}(M)\right)$ is finitely generated. Therefore by [16, Proposition 4.3], $H_{I}^{i}(M)$ is $I$-cofinite. Hence we get that $H_{I}^{i}(M)$ is $I$-cofinite minimax for all $i<t$. Now the assertion follows from Lemma 2.2.

Nhan, in [17, Proposition 5.5], established the following corollary in the case where $R$ is local. The following result provides a slight generalization of [17, Proposition 5.5] and [1, Theorem 2.2].

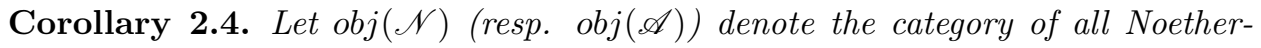
ian (resp. Artinian) R-modules and R-homomorphisms. Let $t$ be a non-negative integer such that $H_{I}^{i}(M) \in \operatorname{obj}(\mathscr{N}) \bigcup \operatorname{obj}(\mathscr{A})$ for all $i<t$. Then the $R$-module $\operatorname{Hom}_{R}\left(R / I, H_{I}^{t}(M)\right)$ is finitely generated. In particular the set $\operatorname{Ass}_{R}\left(H_{I}^{t}(M)\right)$ is finite.

Proof. Apply Theorem 2.3 and the fact that the class of minimax modules includes all Noetherian and Artinian modules.

We are now ready to state and prove the main theorem.

Theorem 2.5. Let $R$ be a Noetherian ring, $M$ a non-zero finitely generated $R$ module and $I$ an ideal of $R$. Let $t$ be a non-negative integer such that $H_{I}^{i}(M)$ is minimax for all $i<t$ and let $N$ be a minimax submodule of $H_{I}^{t}(M)$. Then the $R$-module $\operatorname{Hom}_{R}\left(R / I, H_{I}^{t}(M) / N\right)$ is finitely generated. In particular, the set $\operatorname{Ass}_{R}\left(H_{I}^{t}(M) / N\right)$ is finite.

Proof. In view of Theorem 2.3, $\operatorname{Hom}_{R}\left(R / I, H_{I}^{t}(M)\right)$ is finitely generated. On the other hand, according to Melkersson [16, Proposition 4.3], $N$ is $I$-cofinite. Now, the exact sequence

$$
0 \longrightarrow N \longrightarrow H_{I}^{t}(M) \longrightarrow H_{I}^{t}(M) / N \longrightarrow 0
$$

induces the following exact sequence:

$$
\operatorname{Hom}_{R}\left(R / I, H_{I}^{t}(M)\right) \longrightarrow \operatorname{Hom}_{R}\left(R / I, H_{I}^{t}(M) / N\right) \longrightarrow \operatorname{Ext}_{R}^{1}(R / I, N) .
$$

Consequently $\operatorname{Hom}_{R}\left(R / I, H_{I}^{t}(M) / N\right)$ is finitely generated, as required.

\section{ACKnowledgments}

The authors are deeply grateful to the referee for his/her careful reading and many helpful suggestions on the paper. The authors would like to thank Professor Hossein Zakeri for his reading of the first draft and valuable discussions. 


\section{REFERENCES}

[1] M.P. Brodmann and A.L. Faghani, A finiteness result for associated primes of local cohomology modules, Proc. Amer. Math. Soc. 128(2000), 2851-2853. MR1664309 (2000m:13028)

[2] M.P. Brodmann, Ch. Rotthaus and R.Y. Sharp, On annihilators and associated primes of local cohomology modules, J. Pure and Appl. Algebra 153(2000), 197-227. MR.1783166 (2002b:13027)

[3] M.P. Brodmann and R.Y. Sharp, Local cohomology; an algebraic introduction with geometric applications, Cambridge University Press, Cambridge, 1998. MR 1613627 (99h:13020)

[4] E. Enochs, Flat covers and flat cotorsion modules, Proc. Amer. Math. Soc. 92(1984), 179-184. MR754698 (85j:13016)

[5] A. Grothendieck, Local cohomology, Notes by R. Hartshorne, Lecture Notes in Math., 41, Springer-Verlag, Berlin-New York (1967). MR0224620 (37:219)

[6] A. Grothendieck, Cohomologie locale des faisceaux cohérents et théorèmes de Lefschetz locaux et globaux (SGA2), North-Holland, Amsterdam, 1968. MR0476737 (57:16294)

[7] R. Hartshorne, Affine duality and cofiniteness, Invent. Math. 9(1969/1970), 145-164. MR0257096 (41:1750)

[8] M. Hellus, On the set of associated primes of a local cohomology module, J. Algebra 237(2001), 406-419. MR.1813886 (2001m:13023)

[9] C. Huneke, Problems on local cohomology. Free resolutions in commutative algebra and algebraic geometry, Res. Notes Math., 2, Jones and Bartlett, Boston, MA (1992), 93-108. MR:1165320 (93f:13010)

[10] C. Huneke and R.Y. Sharp, Bass numbers of local cohomology module, Trans. Amer. Soc. 339(1993), 765-779. MR:1124167 (93m:13008)

[11] M. Katzman, An example of an infinite set of associated primes of a local cohomology module, J. Algebra 252(2002), 161-166. MR1922391 (2003h:13021)

[12] G. Lyubezink, Finiteness properties of local cohomology modules (an application of Dmodules to commutative algebra), Invent. Math. 113(1993), 41-55. MR.1223223(94e:13032)

[13] G. Lyubezink, A partial survey of local cohomology. Local cohomology and its applications, Lecture Notes in Pure and Appl. Math., 226, Dekker, New York (2002), 121-154. MR 1888197 (2003b:14006)

[14] T. Marley, The associated primes of local cohomology modules over rings of small dimension, Manuscripta Math. 104(2001), 519-525. MR1836111(2002h:13027)

[15] L. Melkersson, Properties of cofinite modules and application to local cohomology, Math. Proc. Cambridge Philos. Soc. 125(1999), 417-423. MR1656785 (99k:13024)

[16] L. Melkersson, Modules cofinite with respect to an ideal, J. Algebra 285(2005), 649-668. MR2125457 (2006i:13033)

[17] L.T. Nhan, On generalized regular sequences and the finiteness for associated primes of local cohomology modules, Comm. Algebra. 33(2005), 793-806. MR2128412 (2006b:13040)

[18] A.K. Singh, p-torsion elements in local cohomology modules, Math. Res. Lett. 7(2000), 165176. MR:1764314 (2001g:13039)

[19] T. Zink, Endlichkeitsbedingungen für Moduln über einem Noetherschen Ring, Math. Nachr. 64(1974), 239-252. MR0364223 (51:478)

[20] H. Zöschinger, Minimax modules, J. Algebra 102(1986), 1-32. MR853228 (87m:13019)

[21] H. Zöschinger, Über die Maximalbedingung für radikalvolle Untermoduln, Hokkaido Math. J. 17(1988), 101-116. MR928469 (89g:13008)

Department of Mathematics, University of Tabriz, Tabriz, Iran - and - Department of Mathematics, Islamic Azad University-Ardebil Branch, P.O. Box 5614633167, Ardebil, IRAN

E-mail address: bahmanpour@tabrizu.ac.ir

Department of Mathematics, University of Tabriz, Tabriz, Iran - And - School of Mathematics, Institute for Studies in Theoretical Physics and Mathematics (IPM), P.O. Box 19395-5746, TEHRAN, IRAN

E-mail address: naghipour@ipm.ir

E-mail address: naghipour@tabrizu.ac.ir 